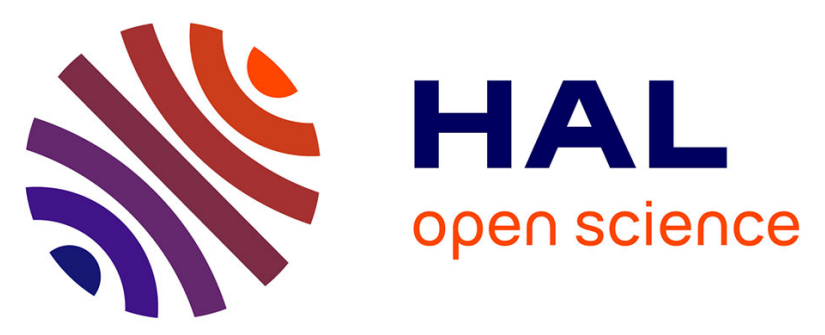

\title{
Exploration de la Physicalité des Widgets pour l'Interaction Basée sur des mouvements de la Tête: le Cas des Menus en Réalité Mixte
}

\author{
Charles Bailly, François Leitner, Laurence Nigay
}

\section{To cite this version:}

Charles Bailly, François Leitner, Laurence Nigay. Exploration de la Physicalité des Widgets pour l'Interaction Basée sur des mouvements de la Tête: le Cas des Menus en Réalité Mixte. Actes de la 32e conférence francophone sur l'Interaction Humain-Machine (IHM'20.21), Apr 2021, Virtual Event, France. pp.11:1-11, 10.1145/3450522.3451326 . hal-03567060

\author{
HAL Id: hal-03567060 \\ https://hal.science/hal-03567060
}

Submitted on 11 Feb 2022

HAL is a multi-disciplinary open access archive for the deposit and dissemination of scientific research documents, whether they are published or not. The documents may come from teaching and research institutions in France or abroad, or from public or private research centers.
L'archive ouverte pluridisciplinaire HAL, est destinée au dépôt et à la diffusion de documents scientifiques de niveau recherche, publiés ou non, émanant des établissements d'enseignement et de recherche français ou étrangers, des laboratoires publics ou privés. 


\title{
Exploration de la Physicalité des Widgets pour l'Interaction Basée sur des mouvements de la Tête : le Cas des Menus en Réalité Mixte
}

\author{
Exploring the Physicality of Widgets for Head-Based Interaction: the Case of Menu in Mixed Reality \\ Charles Bailly ${ }^{1,2}$ \\ ${ }^{1}$ Univ. Grenoble Alpes, CNRS, \\ Grenoble INP, LIG \\ F-38000 Grenoble, France \\ charles.bailly@univ-grenoble- \\ alpes.fr \\ François Leitner ${ }^{2}$ \\ ${ }^{2}$ Aesculap SAS \\ F-38130 Echirolles, France \\ francois.leitner@bbraun.com \\ Laurence Nigay ${ }^{1}$ \\ ${ }^{1}$ Univ. Grenoble Alpes, CNRS, \\ Grenoble INP, LIG \\ F-38000 Grenoble, France \\ laurence.nigay@univ-grenoble- \\ alpes.fr
}

\begin{abstract}
Mixed Reality with a Head-Mounted Display (HMD) offers unique perspectives for head-based interaction with virtual content and widgets. Besides virtual widgets, physical objects can be anchors (mixed widgets) or directly materialised widgets (physical widgets). The physicality (virtual-mixed-physical) of widgets defines a new dimension for Mixed Reality (MR) interaction that extends existing taxonomies of widgets in MR. As a first step to explore this new dimension, we focus on a commonly used widget: a menu. We thus evaluate the performance and usability of head pointing to a virtual, a mixed and a physical menu. Results suggest that pointing to a physical menu was on average $2 \mathrm{~s}$ faster than pointing to a mixed or a virtual menu and preferred by participants. Virtual and mixed menus led to similar performances, but 11 participants over 15 preferred mixed menus over virtual ones. Based on our findings, we provide recommendations (benefits/limitations) for virtual, mixed and physical menus in MR.
\end{abstract}

\section{CCS CONCEPTS}

- Human-centered computing $\rightarrow$ Mixed / augmented reality; Pointing.

\section{KEYWORDS}

Mixed Reality, HMD, Menu, Physicality

\section{RÉSUMÉ}

La réalité mixte avec un casque offre des perspectives uniques pour l'interaction basée sur des mouvements de la tête avec du contenu et des objets d'interaction (widgets) virtuels. Outre les widgets virtuels, les objets physiques peuvent être des ancres (widgets mixtes) ou des widgets directement matérialisés (widgets physiques). La

IHM '20.21, April 13-16, 2021, Virtual Event, France

C 2021 Copyright held by the owner/author(s). Publication rights licensed to ACM. This is the author's version of the work. It is posted here for your personal use. Not for redistribution. The definitive Version of Record was published in Actes de la 32e conférence Francophone sur l'interaction Humain-Machine (IHM'20.21), April 13-16, 2021, Virtual Event, France.

https://doi.org/10.1145/3450522.3451326 physicalité des widgets (virtuelmixte- physique) définit une nouvelle dimension pour l'interaction en Réalité Mixte (RM) qui étend les taxonomies existantes. Pour explorer cette nouvelle dimension, nous nous concentrons sur un widget couramment utilisé : un menu. Les résultats d'une étude expérimentale suggèrent que pointer avec la tête vers un menu physique est en moyenne 2 s plus rapide que pointer vers un menu mixte ou virtuel. Il s'agit de la condition préférée par les participants. Les menus virtuels et mixtes ont conduit à des performances similaires, mais 11 participants sur 15 ont préféré les menus mixtes aux menus virtuels. Sur la base de ces résultats expérimentaux, nous proposons des recommandations (avantages/limites) pour les menus virtuels, mixtes et physiques en RM.

\section{MOTS-CLÉS}

Réalité Mixte, Menu, Physicalité, HMD

\section{INTRODUCTION}

Lorsque les commandes gestuelles ou vocales ne sont pas utilisables, les mouvements de la tête peuvent constituer une technique adaptée pour interagir en Réalité Mixte (RM) avec des lunettes (HMD) [3]. Pour ce faire, un curseur contrôlé avec la tête est souvent utilisé pour pointer vers le contenu virtuel visé. Cette technique a en effet été jugée plus confortable à utiliser et plus précise qu'une technique de pointage basée sur le regard [25, 30]. Ainsi, Bailly et al. ont utilisé un curseur contrôlé avec la tête pour interagir avec un type d'objet interactif (widget) virtuel, qu'est un menu virtuel [3]. Nous adoptons dans nos travaux la même définition de RM que Bailly et al. [3], à savoir un ensemble regroupant la Réalité Augmentée (RA) et la Réalité Virtuelle (RV) [41].

$\mathrm{Au}$ delà de widgets virtuels, nous trouvons le long du Continuum Réalité-Virtualité [36] d'autres types de widgets. Les widgets mixtes comportent une partie physique et une partie virtuelle. S'inscrivant 

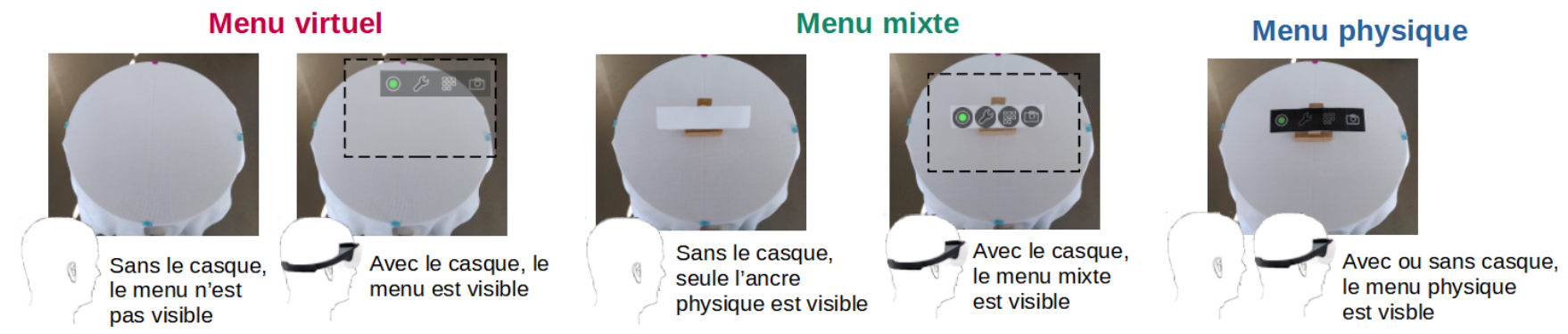

Figure 1: Trois types de menus considérés dans notre étude : menus virtuels, menus mixtes (éléments virtuels attachés à une ancre physique) et menus physiques.

dans la RA tangible [7], des widgets mixtes tels que des menus mixtes [31, 45] sont physiquement manipulés par les utilisateurs. De plus certains widgets peuvent être purement physiques, comme le menu tangible proposé par Ullmer et al. [44]. Ces approches impliquant des widgets mixtes ou physiques se concentrent sur l'interaction tangible en entrée. Notre approche est différente et se focalise sur des propriétés en sortie d'un widget comme sa visibilité sans lunettes de RM ou la flexibilité de l'interface. Nous explorons ainsi une nouvelle dimension pour les widgets : la physicalité.

Indépendamment de la façon d'interagir en entrée avec les widgets, nous considérons 3 types de physicalité pour les widgets :

- Les widgets virtuels ne sont pas spatialement inscrits dans l'environnement physique. Ils sont affichés soit 1) à une position donnée dans le repère de l'écran des lunettes, soit 2) à une position spatiale dans le repère de l'environnement mais sans attache avec l'environnement physique (comme un widget flottant dans les airs). Ces widgets ne sont visibles qu'au travers des lunettes de RM.

- Les widgets mixtes sont basés sur deux composantes : une composante virtuelle et une composante physique. Contrairement aux widgets purement virtuels, ils sont ancrés dans l'environnement physique grâce à leur composante physique. Même sans lunettes de RM, cette ancre physique reste visible (voir Figure 1).

- Les widgets physiques. Ces widgets sont complètement visibles, même en dehors du champ de vision augmenté. En revanche, reposer entièrement sur un objet physique limite leur flexibilité.

L'influence de la physicalité des widgets (widgets virtuels, mixtes ou physiques) sur l'interaction reste à étudier. D'un côté, les widgets physiques sont visibles même en dehors du champ de vision des lunettes et permettent ainsi d'étendre le champ de vision augmenté par définition limité. Néanmoins les widgets physiques limitent la flexibilité de l'interface. D'un autre côté, les widgets virtuels ne sont visibles que dans le champ de vision augmenté et peuvent souffrir de limitations techniques (latence, précision du système de suivi, etc.). Ces différences peuvent avoir un impact sur les performances et les préférences subjectives lors de l'interaction avec un widget, impact qui doit être quantifié. En effet ces considérations sont encore sousexplorées en conception de système de RM alors que des scénarios d'application existent déjà, par exemple en chirurgie augmentée ou en production industrielle [37]. De plus, l'étude des widgets mixtes et physiques aborde certaines limitations critiques des lunettes de RM actuels, dont celle du champ de vision limité.

Dans nos travaux nous considérons un type de widget couramment utilisé : les menus. Dans cette première exploration, nous nous concentrons sur l'aspect perceptuel de la physicalité des menus. Les aspects cognitifs tels que les métaphores d'interaction [32] et la mémorisation de commandes [14] sont en dehors du cadre de nos travaux. La contribution de cet article est une étude comparative des menus virtuels, mixtes et physiques dans le cadre de tâches de pointage en RM réalisées par des mouvements de la tête. L'objectif est d'identifier et de quantifier les avantages et limites des menus physiques par rapport aux menus virtuels et mixtes. Le contexte de la chirurgie augmentée est notre domaine d'application et motive notre étude. Cependant, nous avons mené une étude expérimentale en laboratoire sans condition écologique afin de pouvoir généraliser les résultats à d'autres contextes susceptibles de bénéficier de nos travaux. Après avoir passé en revue les travaux précédents, nous présentons les résultats d'une étude expérimentale menée avec 15 participants pour mesurer l'effet de la physicalité sur les performances et les préférences. Enfin, nous présentons des recommandations et des pistes futures pour la conception de menus mixtes ou physiques.

\section{MOTIVATION : CONTEXTE DE LA CHIRURGIE AUGMENTÉE}

Lors d'une opération, les chirurgiens peuvent être guidés par un système de navigation chirurgicale. Ces systèmes gèrent le flux de travail de la chirurgie (d'où le terme navigation) et guident les chirurgiens en affichant des informations médicales et techniques sur un écran physique distant (jusqu'à $3 \mathrm{~m}$ du patient). Pour interagir avec le système, les chirurgiens peuvent utiliser des techniques de raycasting avec des outils de suivi portatifs spécifiques. Ils peuvent ainsi déclencher des commandes en contrôlant un curseur à l'écran et en sélectionnant des éléments dans des menus à 1 niveau [16].

La RM basée sur un lunettes est un outil puissant pour aider les chirurgiens à effectuer des opérations et les libérer de la dépendance à un unique écran physique distant (Figure 2a). Cependant, la salle d'opération impose des contraintes strictes qui limitent les possibilités d'interaction. Comme expliqué par Bailly et al., les contacts directs avec un objet non stérile et les commandes vocales ne sont pas adaptées à ce contexte [3]. Les techniques d'interaction basées 


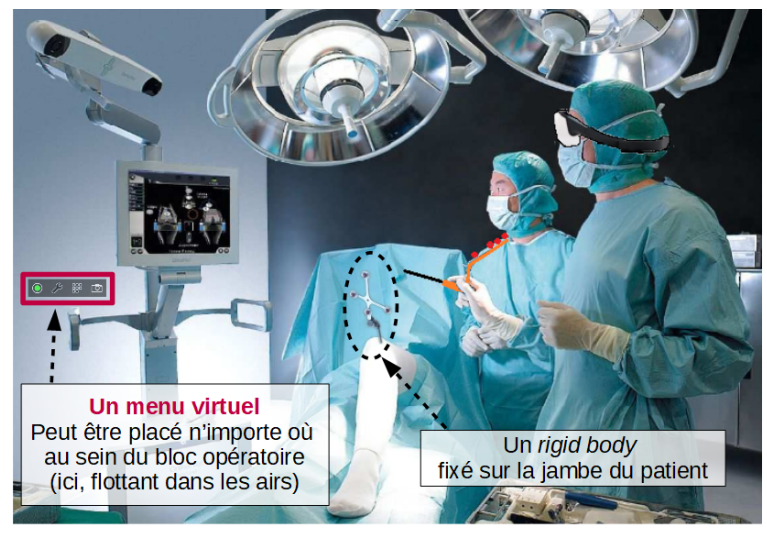

a)

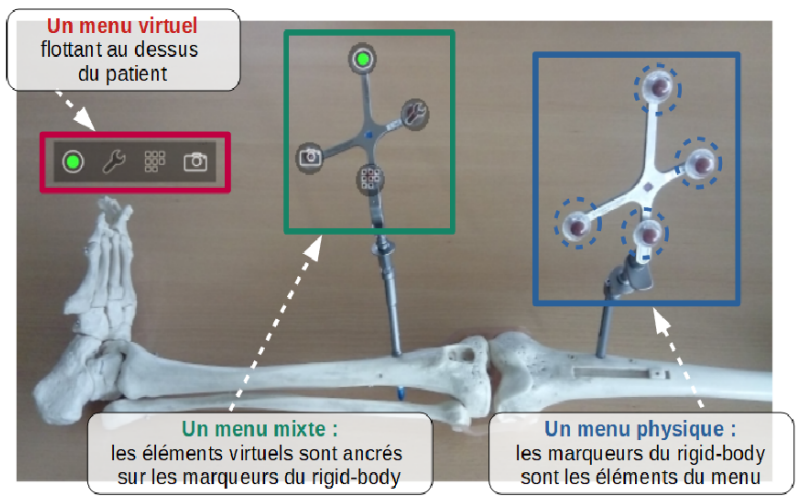

b)

Figure 2: Le bloc opératoire comme environnement interactif. a) Loin du patient : illustration d'un menu virtuel. b) A proximité du patient : illustration d'un menu virtuel, d'un menu mixte dont les éléments virtuels sont ancrés sur des marqueurs et d'un rigid-body utilisé comme menu physique. Images initiales utilisées avec l'autorisation de la société Aesculap.

sur le corps de l'utilisateur [2] posent également problème vis à vis de la stérilité des gants du chirurgien. De plus, laisser autant que possible les mains libres du chirurgien leur permettrait de se concentrer sur les gestes chirurgicaux au lieu d'interrompre leur travail afin de saisir un outil spécifique pour interagir avec le système. Ces raisons nous ont motivés à nous concentrer sur l'interaction basée sur des mouvements de la tête puisque cette technique d'interaction respecte les contraintes de la chirurgie augmentée [3]. La sélection des éléments d'un menu étant une tâche de base courante pour les chirurgiens, nous étudions les tâches de pointage dans un menu.

Avec la RM basée sur des lunettes de RM, l'ensemble du bloc opératoire peut devenir un environnement interactif avec plusieurs types de menus, comme le montre la Figure 2. Des menus virtuels peuvent être placés tout autour du chirurgien (Figure 2a). Plus proche du patient où de fortes contraintes de stérilité s'appliquent, des menus physiques et mixtes sont également possibles. Au début d'une opération, les chirurgiens fixent des objets métalliques appelés rigid-bodies sur le corps du patient. Ces objets comprennent 4 marqueurs suivis par une caméra (Figure 2). Les rigid-bodies permettent au système de suivre la position du patient et ainsi de guider les chirurgiens pendant l'opération. La fixation des rigid-bodies fait déjà partie de la procédure classique d'une chirurgie orthopédique assistée par ordinateur. Pour certains systèmes de navigation chirurgicale, les chirurgiens peuvent déclencher des commandes en pointant vers des marqueurs d'un rigid-body avec un outil physique. Dans ce cas, les éléments de menu sont physiques et une temporisation (dwell time) est utilisée comme mécanisme de validation. Les rigid-bodies peuvent ainsi être utilisés comme menus physiques ou mixtes, comme le montre la Figure $2 b$.

L'étude de l'impact de la physicalité des widgets sur l'interaction est pertinente dans le contexte de la chirurgie augmentée. Nous avons implémenté des menus virtuels, mixtes et physiques dans un prototype basé sur un logiciel de navigation chirurgicale existant appelé OrthoPilot [1]. Dans cet article, la chirurgie augmentée est utilisée comme un domaine d'application illustratif, mais d'autres domaines tels que la maintenance de machines industrielles en RM pourraient également bénéficier de notre étude.

\section{3 ÉTAT DE L'ART}

\subsection{Physicalité des cibles}

Jansen et al. ont étudié l'impact de la physicalité d'un objet dans le domaine de la visualisation d'information [26]. Les auteurs ont observé que l'utilisation d'un objet physique pour la visualisation 3D conduisait à de meilleures performances pour les tâches de recherche d'informations que l'utilisation d'une visualisation à l'écran [26]. Un facteur notable souligné par les auteurs pour expliquer cette différence de performance est le réalisme visuel des objets physiques. Les auteurs ont mentionné plusieurs signaux visuels tels que les indices stéréoscopiques, les indices d'accommodation et la résolution qui peuvent avoir eu un impact sur l'interaction. Cette notion de réalisme visuel soulève plusieurs questions pour la RM. Des problèmes de perception comme la fidélité des couleurs, la stéréoscopie et les conflits de vergence-accommodation représentent encore des défis techniques en RM [29]. Le réalisme visuel des cibles virtuelles et mixtes peut ainsi influencer les performances des tâches de pointage.

\subsection{Physicalité des menus}

De nombreux travaux dédiés à l'interaction avec des menus en RM se concentrent sur l'interaction tangible avec ces menus, également appelée réalité augmentée tangible (RA). Selon Billinghurst et al., "Les interfaces de RA tangibles sont celles dans lesquelles 1) chaque objet virtuel est lié à un objet physique et 2) l'utilisateur interagit avec des objets virtuels en manipulant les objets physiques correspondants" [7]. Adoptant ce paradigme d'interaction, plusieurs menus mixtes ont été proposés. Les Shake menus sont des menus radiaux virtuels centrés sur une tuile physique [45]. L'utilisateur secoue la tuile pour activer les menus. Lee et al. ont proposé un cube tangible dont les faces peuvent être physiquement tournées pour sélectionner des éléments virtuels sur un menu en anneau attaché au cube [31]. 
Cela correspond à un menu mixte puisqu'il combine des éléments virtuels liés aux faces d'un objet physique. Les menus complètement physiques sont beaucoup moins courants. Ullmer et al. ont proposé le concept de menus tangibles : des liens numériques (comme des commandes "ouvrir" et "sauvegarder") sur des surfaces physiques (papier, bois, plastique) [44].

Au-delà du domaine de la RM, de nombreux travaux sur la physicalité des menus adoptent les principes des interfaces utilisateur tangibles (TUI), tels que définis par Ishii [23]. Notre approche diffère de ces travaux car nous nous concentrons uniquement sur l'incarnation physique des menus et ne nous intéressons pas ici aux modalités d'interaction en entrée impliquant la manipulation d'objets physiques. Le terme tangibilité n'est donc pas adapté à notre étude. Dans le reste de l'article, nous utilisons le terme physicalité pour la physicalité (des widgets) et nous désignons par menu physique un menu avec une représentation physique.

Utiliser des menus physiques plutôt que des menus mixtes ou virtuels peut sembler évident au premier abord. Les menus physiques ne souffrent pas de limitations techniques telles qu'un faible champ de vision, la latence ou un manque de précision du système de suivi des mouvements. Ils semblent donc idéaux comme cibles de pointage. Par ailleurs, les menus physiques sont directement et entièrement visibles dans la vision périphérique, ce qui peut aider les utilisateurs à mieux planifier leurs mouvements de tête. Cela peut impacter en particulier la phase préliminaire de pointage dans le modèle de Meyer et al. [34].

Cependant, les menus physiques présentent aussi des limitations principalement en terme de flexibilité par rapport aux menus virtuels :

- Les menus physiques reposent sur des objets physiques, dont l'ajout peut ne pas convenir à tous les contextes. Par exemple, ils ne sont pas adaptés aux environnements encombrés tels que les salles des machines ou les véhicules [19] et impliquent un environnement prédéfini n'autorisant pas l'interaction mobile [38].

- Même lorsqu'ils conviennent, les menus physiques ont un impact sur la spatialité de l'interface utilisateur car ils reposent sur des positions fixes spécifiques. Contrairement aux menus virtuels, ils ne peuvent pas être placés n'importe où Le contrôle de l'utilisateur sur ces menus peut être limité.

- Les menus physiques ont un coût en ce qui concerne leur fabrication, leur mise en place et leur mise à jour.

De plus les transitions fréquentes de focus visuel peuvent avoir un impact sur l'interaction avec des menus physiques en RM. Les conflits de vergence-accommodation sont connus pour avoir un impact négatif sur les performances et augmenter la fatigue visuelle [21, 29]. La nature de la tâche à effectuer (par exemple, la fréquence des transitions visuelles, la durée de la tâche et la charge cognitive) et les caractéristiques de l'environnement (par exemple la complexité, la congestion, la physicalité et la position des objets) peuvent influencer les performances et le confort. Par conséquent, supposer que l'utilisation de menus physiques est toujours la meilleure approche n'est au final pas si évident.

\subsection{Positionnement de notre étude}

L'analyse de l'impact des cibles non virtuelles sur les tâches de pointage en RM a été mentionnée comme un travail futur par Henze et al. dans leur étude sur la visualisation de cibles hors écran dans des cartes augmentées [20]. Plus récemment, Bailly et al. ont proposé et comparé trois techniques basées sur les mouvements de la tête pour pointer vers un menu en RM avec un casque [3]. Les techniques impliquaient des menus virtuels ainsi que des menus mixtes. Les auteurs ont également proposé un espace de conception (Figure 3) organisé selon 2 facteurs de conception : 1) l'élément d'interaction contrôlé par l'utilisateur et 2) la physicalité de la cible [3].

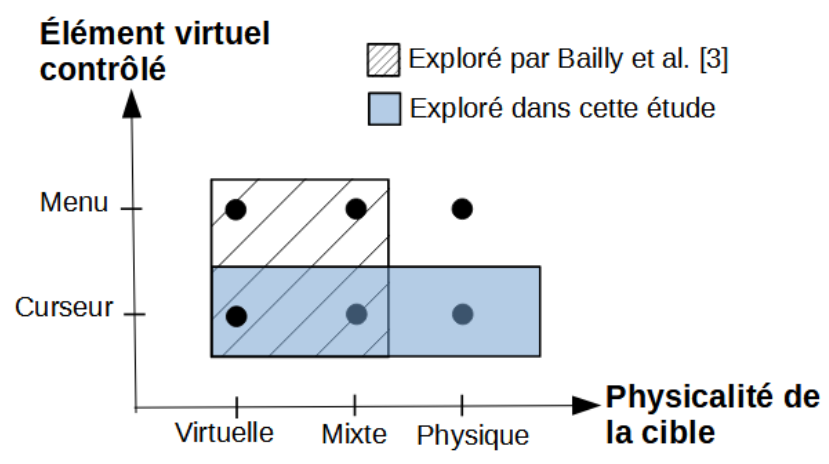

Figure 3: Parties de l'espace de conception explorées dans notre étude par rapport aux travaux de Bailly et al. [3].

$\mathrm{Au}$ regard de cet espace de conception, nous considérons un curseur contrôlé par la tête pour interagir avec des menus virtuels, mixtes et physiques, tandis que l'étude de Bailly et al. se concentre uniquement sur des menus virtuels et mixtes [3]. De plus, la conception de notre menu mixte est différente puisque les objets physiques utilisés comme ancres ont la même forme rectangulaire que les menus virtuels, contrairement à [3]. Par ailleurs, notre protocole expérimental implique également une tâche de distraction. Cette tâche a été créée : 1) pour augmenter la fréquence des transitions de focus visuel et 2) pour augmenter la charge cognitive des participants et refléter la charge cognitive d'utilisateurs mentalement occupés. Enfin, notre objectif est de quantifier la différence de performance entre les menus virtuels, mixtes et physiques car cet aspect est absent des travaux précédents.

\section{CONCEPTION ET MISE EN EUVRE}

\subsection{Conception des menus}

Nous considérons dans cette étude uniquement des menus à 1 niveau avec 4 éléments. Ce choix de conception a été motivé par la similitude avec les menus des systèmes de navigation chirurgicale existants. Notre étude exclut donc le cas des menus hiérarchiques à plusieurs niveaux, impactant le facteur d'applicabilité comme exposé dans la taxonomie de menus de Bailly et al. [4]. Pour une comparaison valide des menus virtuels, mixtes et physiques, notre objectif de conception était de rendre les menus aussi similaires que possible. Pour ce faire, des rectangles de papier (12,5 $\mathrm{cm}$ de long, 3,5 $\mathrm{cm}$ de haut) ont été utilisés comme ancres physiques pour indiquer 
les emplacements des menus. Les ancres étaient fixées à un panneau blanc à l'aide de ruban adhésif transparent et fabriquées à partir de papier vert pour s'assurer que les ancres étaient clairement visibles. Les ancres physiques correspondaient ainsi à la forme rectangulaire des menus virtuels. L'objectif était d'obtenir des ancres neutres, sans lien sémantique avec le menu ni métaphore particulière. Les éléments des menus étaient des lettres. Pour les menus physiques, les lettres étaient écrites sur des feuilles de plastique transparentes à l'aide d'un marqueur noir. Ces feuilles de plastique transparentes étaient fixées et superposées à des ancres. Les éléments physiques étaient un peu plus petits que les ancres physiques $(12 \mathrm{~cm}$ de long, 3 $\mathrm{cm}$ de haut). Globalement les trois types de menus implémentés ont la même taille visuelle, et la figure 4 les présente pour comparaison.

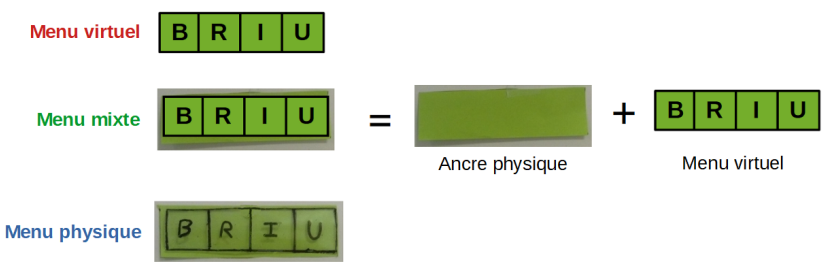

Figure 4: Trois types de menus réalisés : menu virtuel, mixte et physique.

Les menus virtuels, mixtes et physiques constituant des conditions dans l'étude expérimentale menée ont été implémentés de la même manière. Pour chaque condition, le participant pointait des menus virtuels dont les positions prédéfinies étaient connues du système. Dans la condition Menus physiques, les menus virtuels n'étaient pas affichés à l'écran. Ils étaient néanmoins présents et alignés avec les emplacements des menus physiques. Lors des tâches de pointage, le système se basait toujours sur ces menus virtuels (masqués ou non) pour vérifier si le participant avait correctement sélectionné l'élément cible. Ainsi, les trois types de menus étaient implémentés de la même manière puisque seule la visibilité des menus virtuels différait.

\subsection{Technique d'interaction}

Comme le montre la Figure 3, nous nous concentrons sur des techniques basées sur le contrôle d'un curseur pour sélectionner des éléments d'un menu. Pour ce faire, nous avons ré-implémenté la technique Head-Controlled Cursor décrite dans [3]. Un curseur virtuel en forme de croix est affiché au centre du champ de vision des lunettes. Les utilisateurs doivent viser un élément d'un menu en orientant leur tête et en alignant le curseur sur l'élément cible du menu. La taille visuelle du curseur est de $1 \times 1 \mathrm{~cm}$ tandis que la taille visuelle d'un élément de menu (en forme de carré) est de $3 \times 3 \mathrm{~cm}$. La sélection d'un élément de menu est confirmée en appuyant sur un bouton de la télécommande des lunettes de RM (voir Section 4.3 et Figure 5). Un tel mécanisme de confirmation pourrait être remplacé par une pédale (dispositif couramment utilisé en chirurgie). Utiliser la tête plutôt que le regard pour pointer est plus confortable et précis $[25,30]$. De plus, pointer avec la tête permet d'interagir sans utiliser les mains. Ce type de technique est disponible dans certains casques actuellement commercialisés comme l'HoloLens 1 et 2 [35]. Ces raisons ont motivé notre choix de l'utiliser comme technique de référence.

\subsection{Implémentation technique}

Nous avons utilisé les lunettes de RM Epson Moverio BT-300, fonctionnant sous Android 5.1. Ces lunettes ont un champ de vision de 23 degrés en diagonale (environ $20^{\circ}$ à l'horizontale), une résolution de $1280^{*} 720$ pixels et a l'avantage d'être léger. Ces lunettes sont également reliées en filaire à une télécommande, que nous avons utilisé dans notre étude comme périphérique d'entrée lors de l'interaction avec le système. Trois marqueurs passifs ont été fixés sur les lunettes afin de suivre sa position avec un ensemble de cinq caméras Flex3. Ces caméras sont fixées au plafond et contrôlées par le logiciel Tracking Tools (version 2.3.1) fonctionnant sur un ordinateur portable distant. Un script Python envoie la position de la tête calculée aux lunettes via Wifi. Notre logiciel fonctionnant sur les lunettes utilise cette position de la tête (emplacement et orientation) pour afficher la vue correspondante. Ce logiciel est basé sur OpenGL ES 2.0 pour afficher du contenu virtuel en 3D côte à côte. Globalement, le système mis en œuvre est capable de maintenir 30fps. Une moyenne de $24 \mathrm{~ms}$ est nécessaire pour obtenir l'estimation de la pose de la tête : $10 \mathrm{~ms}$ pour les caméras, $4 \mathrm{~ms}$ pour les calculs de pose et $10 \mathrm{~ms}$ pour envoyer la pose aux lunettes via Wifi.

\section{5 ÉTUDE EXPÉRIMENTALE}

15 participants volontaires ( 2 femmes, 13 hommes) âgés de 19 à 45 ans $(s d=6,6)$ ont été recrutés pour participer à notre étude sur la comparaison des menus virtuels, mixtes en physiques en RM. Comme indiqué dans notre questionnaire préliminaire, ils étaient tous novices en RM et seuls 3 d'entre eux avaient déjà essayé de porter un casque de RM auparavant. Les participants pouvaient porter des lunettes de vue sous les BT-300 si nécessaire. Comme notre protocole impliquait une tâche de calcul mental (tâche de distraction), nous nous sommes assurés que tous les participants avaient une bonne connaissance des mathématiques usuelles.

\subsection{Installation expérimentale}

Les participants se tenaient derrière une table carrée grise $(81 \mathrm{~cm}$ de long, $73 \mathrm{~cm}$ de haut) représentant une zone de travail. Un panneau blanc (102 cm de long, $125 \mathrm{~cm}$ de haut) a été placé verticalement derrière la table et surélevé du sol. La zone de panneau résultante au-dessus de la table (102 cm de long, $74 \mathrm{~cm}$ de haut) a été utilisée comme zone principale pour les tâches expérimentales (c'est-à-dire où les menus apparaissent). L'installation est présentée à la Figure 5.

\subsection{Tâches}

L'expérimentation était divisée en deux types de tâches : les tâches de distraction et les tâches de pointage. Les tâches de distraction étaient basées sur le raisonnement mathématique et la mémorisation. Pour les tâches de pointage, les participants devaient sélectionner un élément de menu. Au cours de l'expérimentation, chaque étape comprenait deux tâches de distraction suivies d'une tâche de pointage. 


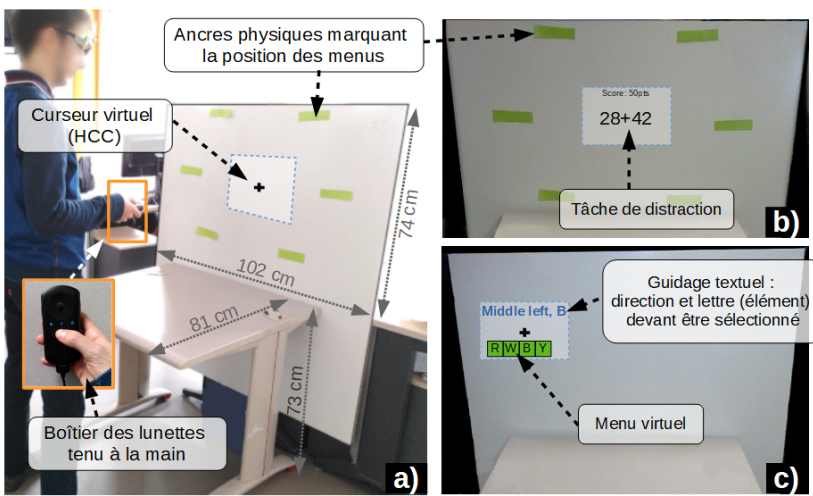

Figure 5: Installation expérimentale pour l'étude. a) Participant devant l'espace de travail. b) Tâche de distraction. c) Tâche de pointage.

5.2.1 Tâche de distraction. Le raisonnement mathématique est une tâche couramment utilisée pour augmenter la charge cognitive des participants lors des expériences [8, 17, 22]. Plusieurs variantes de ce type de tâche peuvent être trouvées dans la littérature, y compris le calcul mental $[8,22]$ et la comparaison des nombres [17]. La mémorisation peut également être trouvée dans d'autres types de tâches comme le jeu de la $N^{e m e}$ lettre dans une étude récente d'Evain et al. [12]. Dans notre expérimentation, la tâche de distraction combinait addition, comparaison et mémorisation de nombres. Notre objectif était d'augmenter uniquement la charge cognitive des participants. Aucune habileté motrice n'était requise. En effet, nous ne cherchons pas ici à simuler une tâche chirurgicale mais simplement une tâche principale abstraite afin de pouvoir généraliser les résultats. Nous voulions une tâche non liée à la chirurgie demandant une attention visuelle au centre du champ de vision augmenté initial.

La tâche de distraction était présentée aux participants comme tâche principale afin qu'elle soit effectuée avec toute l'attention requise. L'objectif était de marquer autant de points que possible en répondant correctement à une succession de tâches arithmétiques. Les participants devaient calculer mentalement l'addition de nombres à 2 chiffres affichés au centre de la zone de travail et compris entre 10 et 50 . Ce résultat devait être comparé au résultat de l'addition précédente et la réponse devait être donnée grâce au pavé tactile de la télécommande des lunettes Epson Moverio BT-300. Si le résultat $R_{N}$ de l'addition courante était supérieur au résultat de l'addition précédente $R_{N-1}$, il fallait appuyer sur la flèche supérieure du pavé tactile. Sinon, il fallait appuyer sur la flèche du bas. Deux additions consécutives ne donnaient jamais le même résultat. Les participants devaient mémoriser le résultat courant d'une addition à l'autre et une tâche de pointage pouvait être effectuée entre deux additions.

5.2.2 Tâche de pointage. Pour les tâches de pointage, les participants devaient sélectionner un élément dans un menu à un niveau de 4 éléments en déplaçant la tête. Ce choix de conception a été motivé par la similitude avec les menus des systèmes de navigation chirurgicale existants tels que l'OrthoPilot ${ }^{\circledR}[1]$. Chaque élément de menu était identifié par une lettre aléatoire unique. Pour sélectionner un élément, les participants devaient suivre les instructions textuelles apparaissant en haut de l'écran des lunettes. Ces informations affichées comprenaient la position du menu et l'élément de menu (par exemple, au milieu à gauche, B), comme le montre la figure $5 \mathrm{c}$. Les participants devaient ensuite bouger la tête pour aligner un curseur virtuel au centre de l'écran avec l'élément de menu correspondant. La sélection était validée à l'aide du bouton central de la télécommande des lunettes. Les participants avaient pour consigne d'aller le plus vite possible sans faire d'erreur. En cas de sélection incorrecte, les participants devaient continuer avec la cible actuelle jusqu'à la sélectionner.

Nous nous sommes inspirés du peephole pointing et d'un pointage en deux phases (pointer vers le menu, puis vers un élément du menu) [39]. Pour équilibrer la première phase, nous nous sommes inspirés d'une tâche de pointage ISO 9241-9 classique [24] pour positionner les menus. Six menus étaient placés sur le panneau au-dessus de la table pour former un motif circulaire, comme le montre la Figure 5. Les participants commençaient par deux tâches de distraction au centre du cercle, puis devaient pointer vers un élément de l'un des 6 menus du cercle. Tous les menus se trouvaient en dehors du champ de vision augmenté initial au centre du cercle. Un tel placement est lié à notre objet d'étude : étudier dans quelle mesure le fait de ne pas voir la position du menu (menu virtuel) ou de voir sa position mais pas ses éléments (menu mixte) impacte l'interaction par rapport à des menus physiques. Nous cherchons à étendre le champ augmenté limité par la physicalité des menus. La distance angulaire pour atteindre le centre des menus à partir de la position centrale était constante et proche de 33 degrés.

L'ordre des cibles (menus et éléments de menu) était aléatoire. Au début de la condition Menus mixtes, les participants étaient informés que l'emplacement des cibles possibles était indiqué par les repères verts. Pour la condition Menus physiques, les participants étaient encouragés à cibler les lettres physiques. Pour les menus virtuels, le panneau blanc était retourné par le superviseur afin de masquer les ancres et menus physiques. L'indice de difficulté des cibles a été fixé entre 4,1 et 4,2 selon l'élément de menu ciblé. Cet ID a été calculé en utilisant la formulation de Wingrave et Bowman de la loi de Fitts [46]. D'après Soukoreff et Mackenzie, nos tâches de pointage sont de difficulté moyenne [40].

\subsection{Conception de l'étude}

L'expérience était divisée en 3 blocs de 30 pointages, un bloc par condition : Menus virtuels, Menus mixtes et Menus physiques. Au total, il y avait 3 conditions $\mathrm{x} 6$ positions de menu $\mathrm{x} 5$ répétitions, soit 90 tâches de pointage par participant. L'ordre des blocs était équilibré grâce à un carré latin standard. Au début de l'expérience, les participants pouvaient d'abord ajuster l'effet stéréo des lunettes si nécessaire. Ensuite, ils pouvaient répéter la tâche de distraction autant qu'ils le souhaitaient jusqu'à être certain de bien avoir compris le principe. Avant chaque bloc, 12 étapes d'entraînement étaient imposées pour permettre aux participants de se familiariser avec la physicalité du menu. Cette phase d'entraînement était similaire aux phases réelles : les participants alternaient tâches de distraction et tâche de pointage. À la fin d'un bloc, les participants pouvaient faire une pause avant de répondre à un questionnaire Raw-TLX 
[18] pour recueillir leurs opinions sur la session effectuée. Un questionnaire final était remis à la fin de l'expérience pour obtenir des commentaires sur la comparaison de toutes les conditions.

Notre objectif était d'éviter tout effet d'apprentissage sur les positions des menus. Pour se faire, un guidage textuel était affiché dès le début de chaque tâche de pointage, comme expliqué auparavant. De plus, l'expérience comprend un petit nombre de menus et une session d'entraînement conséquente. L'objectif était de permettre aux participants de se familiariser avec les tâches et les 6 menus, ce qui nous a permis d'utiliser une conception expérimentale intra-sujet $[15,33]$.

\subsection{Mesures}

Le temps de sélection et les nombres d'erreur ont été enregistrés à chaque pointage. En cas de sélection invalide, la distance jusqu'à l'élément cible du menu était également enregistrée. De plus, les questionnaires Raw-TLX donnés étaient basés sur une échelle de Likert à 5 éléments. Le questionnaire final comprenait des questions sur les performances subjectives et les préférences concernant les 3 conditions physiques du menu. Les préférences des participants et l'utilité perçue des ancres étaient également discutées lors d'un entretien semi-structuré.

\subsection{Résultats}

Pour l'analyse statistique, une ANOVA à mesures répétées (avec $\alpha$ $=0,05)$ a été le principal outil statistique utilisé. Des tests post hoc ont été réalisés en utilisant des t-tests pairés et des corrections de Bonferroni. Comme suggéré par Dragicevic [10], une transformation logarithmique a été appliquée aux données lorsque cela était pertinent (c'est-à-dire pour le cas d'une asymétrie positive dans les mesures de temps). Lorsqu'une ANOVA n'était pas appropriée, nous avons utilisé un test de Friedman suivi d'un test post hoc de Wilcoxon avec des corrections de Bonferonni. Dans toutes les figures, les barres d'erreur sont des intervalles de confiance (IC) à $95 \%$.

5.5.1 Temps de sélection d'un élément d'un menu. Le temps de sélection moyen pour les menus virtuels et les menus mixtes est proche de 6s. Pour les menus physiques ce temps est pratiquement réduit de 2 s, comme illustré à la Figure $6 \mathrm{a}$. Cette tendance est soutenue par l'analyse statistique, qui a fourni de bonnes preuves que la condition Menus physiques était différente de la condition Menus virtuels $(p=0.0021)$ et de la condition Menus mixte ( $p=$ $0.0027)$. Aucune preuve de différence n'a été trouvée entre les menus virtuels et mixtes $(p=1)$.

6 secondes est un temps relativement long pour sélectionner un élément d'un menu avec un curseur contrôlé par la tête. Dans l'étude menée par Bailly et al [3], le temps de sélection moyen ne dépassait pas 4 secondes. Tout d'abord l'indice de difficulté de la tâche de pointage était légèrement plus élevé dans notre étude (entre 4.1 et 4.2 au lieu d'environ 3.2 dans l'étude de Bailly et al. [3]). De plus, les participants avaient à réaliser une tâche secondaire pour augmenter de manière importante leur charge cognitive, contrairement à l'étude de Bailly et al. [3]. Comme détaillé dans la section sur les retours qualitatifs, la plupart des participants ont jugé les tâches mentalement exigeantes. La charge cognitive due à la difficulté d'une tâche mathématique peut avoir un impact important sur les performances [11, 17]. Nous supposons que la tâche de distraction a empêché les participants de se concentrer sur la tâche de pointage et d'atteindre une vitesse optimale.

Enfin, nous n'avons pas observé de différence de performance entre les différentes positions de menu et entre les éléments des menus. Une telle observation va à l'encontre de certains travaux de la littérature sur les rotations de la tête [42]. Néanmoins, cela peut s'expliquer dans notre étude par une amplitude de mouvement de la tête fixe relativement faible.

5.5.2 Transfert asymétrique de compétences. Les résultats précédents sur les temps de sélection moyens doivent être nuancés par le transfert asymétrique de compétences observé dans notre étude. Nous avons vérifié si l'ordre des conditions pouvait affecter les performances. Comme expliqué ci-dessus, nous nous sommes assurés que la position des menus était bien comprise par les participants avant qu'ils ne commencent les essais et nous avons affiché des indications textuelles sur l'emplacement du menu. Cependant, même avec des positions de menu parfaitement connues au début des essais, nous avons tout de même observé un transfert de compétences asymétrique, comme illustré par les figures $6 \mathrm{~b}, \mathrm{c}$ et $\mathrm{d}$. Ce phénomène a été partiellement corroboré par notre analyse statistique. Comme suggéré par Cockburn et al. [9], nous avons vérifié l'effet principal de l'ordre des conditions sur les temps de sélection, et avons trouvé une preuve d'effet moyenne $(p=0,076)$.

Nous avons trouvé des preuves moyennes que les participants qui ont commencé avec des menus virtuels se sont améliorés lorsqu'ils ont utilisé des menus mixtes par la suite (Figure $6 \mathrm{~b}, p=0,077$ ). Cette tendance était beaucoup plus claire des menus virtuels aux menus physiques $(p=0,0026)$. Lorsque les participants ont commencé avec des menus mixtes, le phénomène est plus subtil (Figure 6c), et nous n'avons observé qu'une preuve de différence moyenne entre la première condition (Menus mixtes) et la seconde condition (Condition Menus physiques, $p=0,14)$. Cependant, nous avons observé une tendance complètement différente pour les participants qui ont commencé avec des menus physiques. Dans ce cas, la performance globale des participants avec des menus virtuels et mixtes était beaucoup plus stable une fois qu'ils ont utilisé les menus physiques (Figure 6d, aucune preuve de différence trouvée, $p=1$ ).

Par conséquent, il semble qu'il y ait eu un transfert asymétrique de compétences entre les conditions. Les participants ont pu transférer les compétences acquises à l'aide de menus physiques vers d'autres conditions. Nous avons observé des tendances similaires lors de la suppression des 10 premiers essais de chaque condition pour nous assurer qu'aucun effet d'apprentissage sur les positions de menu n'était impliqué. Néanmoins, malgré le transfert apparent de compétences, les menus physiques semblent toujours plus rapides à utiliser.

5.5.3 Erreurs. Très peu d'erreurs ont été commises lors des essais, indépendamment de la physicalité des menus. Nous avons trouvé de bonnes preuves de différence pour les menus physiques, avec lesquels les participants ont commis un peu plus d'erreurs $\left(\chi^{2}=\right.$ $13,849, p=0,001)$. Plus précisément, le test post hoc de Wilcoxon corrigé par Bonferroni a suggéré que la condition Menus Physiques était différente de la condition Menus Mixtes $(p=0.011)$ et de la 


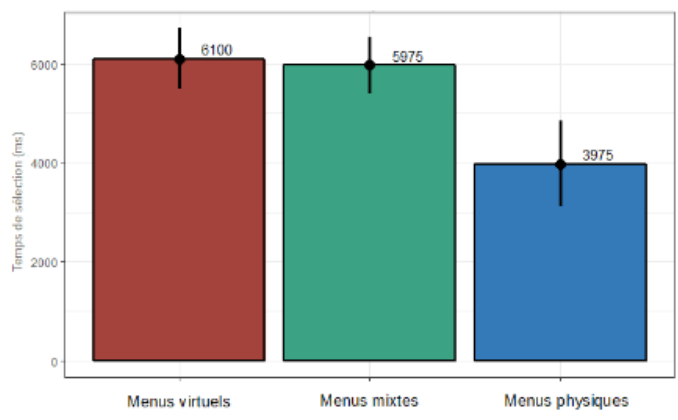

a)

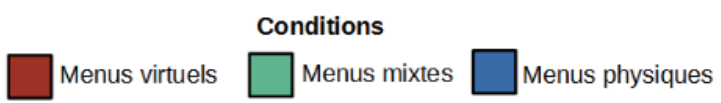

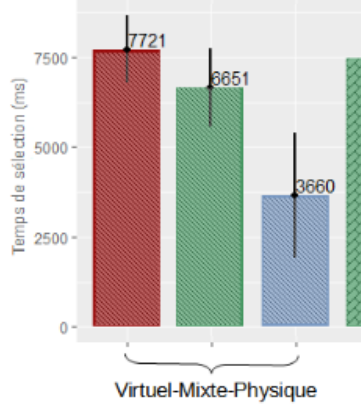

b)

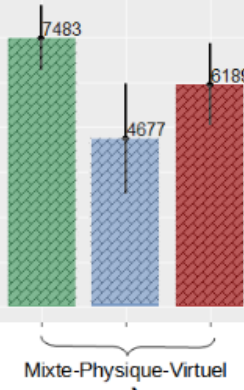

c)

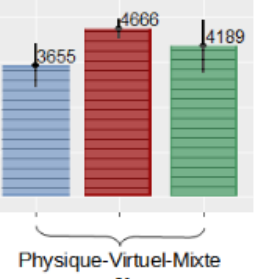

d)

Ordre des conditions

Virtuel-Mixte-Physique Mixte-Physique-Virtuel

Physique-Virtuel-Mixte

Figure 6: Temps de sélections moyens (ms). a) Pour chaque type de menu. b), c) et d) : Transfert asymétrique de compétences.

condition Menus Virtuels $(p=0,024)$. Cependant, l'ordre de grandeur de cette différence reste néanmoins très faible. Comme le montre la Figure 7, le taux d'erreur moyen pour les menus physiques est aux alentours de $0.75 \%$.

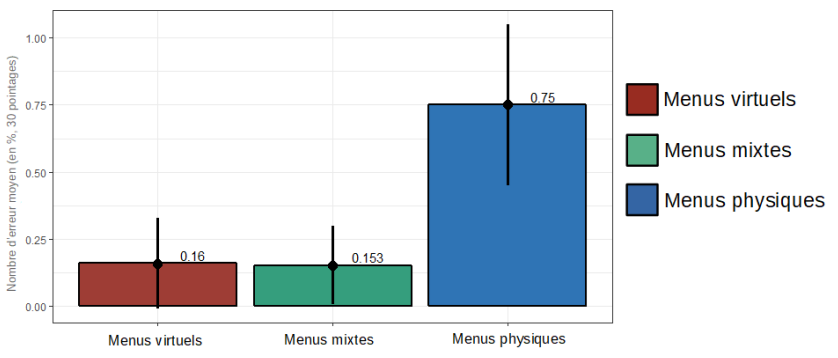

Figure 7: Étude 3 : Taux d'erreur moyen (en \%) sur 30 pointages.

5.5.4 Retours qualitatifs. Les questionnaires Raw-TLX donnés à la fin de chaque condition ont confirmé que les participants ressentaient une charge cognitive importante pendant les tâches. Pour chaque condition, une majorité de participants ont répondu que la tâche était au moins Plutôt exigeante sur le plan mental : la charge mentale était généralement classée $4 / 5$ ou $5 / 5$ sur l'échelle de Likert à 5 points. Des réponses similaires ont été données pour l'effort subjectif requis pour réaliser les tâches, qui a été perçu comme au moins assez élevé par la plupart des participants (indépendamment de la condition). Ces résultats ont confirmé que les tâches de distraction affectaient efficacement la charge cognitive des participants pendant les essais.

Le questionnaire final demandait aux participants de classer les 3 conditions selon 5 critères : facilité d'utilisation, rapidité, précision, fatigue et condition favorite. Les résultats de ce classement sont particulièrement clairs car la condition Menus physiques a été systématiquement préférée aux deux autres conditions, comme le montre la figure 8 . Ce résultat est cohérent avec les résultats quantitatifs. La condition Menus mixtes a été largement classée au second rang, et la condition Menus virtuels a été largement jugée comme la pire. Cette tendance claire entre Menus mixtes et Menus virtuels est également intéressante puisqu'elle n'est pas reflétée par les résultats quantitatifs.
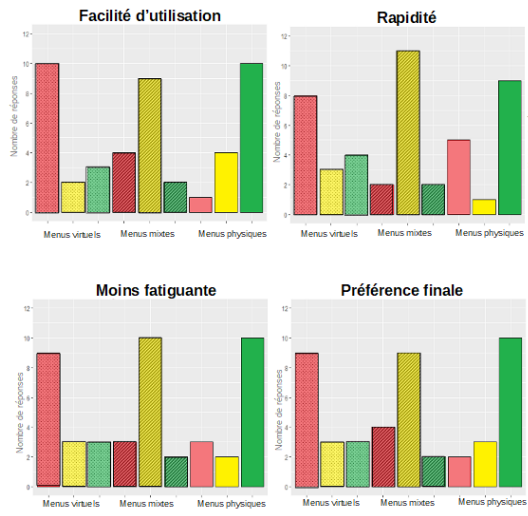

Préférence finale
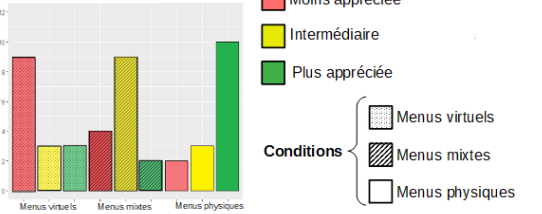

\section{FIGURE 8: Retours qualitatifs pour chaque condition.}

Les entretiens semi-structurés indiquent que les menus physiques ont été globalement jugés plus faciles à utiliser car les éléments de menu étaient toujours visibles et parfaitement stables. 10 participants sur 15 ont trouvé que les ancrages physiques (la partie physique des menus mixtes) étaient au moins Plutôt utiles. La plupart de ces 10 participants ont indiqué que les ancres les ont aidés à voir les emplacements des menus avant de commencer la tâche de pointage. 4 autres participants n'ont pas jugé les ancres physiques utiles, expliquant que l'information textuelle affichée était suffisante pour connaître l'emplacement du menu.

Enfin, deux opinions contradictoires sur les ancres physiques étaient particulièrement intéressantes. Un participant a expliqué 
"Pour Mixte, j'ai utilisé les ancres pour aller dans le menu, j’ai ensuite cherché la lettre (élément de menu), et enfin j'ai regardé l'ancre lors de la validation de la sélection". Autrement dit, ce participant s'est concentré sur l'ancre physique avant de valider la sélection de l'élément virtuel du menu. Cela a été rendu possible grâce à la forme des ancres, qui était similaire à la forme des éléments virtuels. Au contraire, un autre participant n'a pas apprécié et a jugé dérangeant la similitude entre l'ancre et les éléments du menu, expliquant que les ancres des menus mixtes étaient comme des "doublons". Le participant a ensuite évoqué l'idée d'ancres physiques différentes des éléments virtuels du menu, sans superposition complète. Ce participant aurait préféré de petits repères physiques montrant simplement la position des menus au lieu d'une ancre qui couvre l'ensemble des éléments du menu.

\section{LEÇONS ISSUES DE L'ÉTUDE EXPÉRIMENTALE}

L'implication principale de cette étude est que les menus physiques peuvent surpasser les menus mixtes et virtuels. La condition Menus physiques est également la condition préférée par les participants et leur permettait de transférer des compétences acquises à l'aide de menus physiques vers d'autres types de menus. Pour les menus physiques, le pointage est en une seule phase : voir la cible finale (l'élément du menu physique) et non juste une position globale (l'ancre du menu mixte) a pu affecter la planification et donc la réalisation du geste balistique [34].

Ces résultats expérimentaux et les enseignements décrits cidessous sont néanmoins à nuancer par rapport aux limitations de notre étude. En effet, dans cette première exploration de la physicalité des menus, nous avons considéré des menus simples à un seul niveau. Par ailleurs, la faible taille du champ de vision augmenté $\left(23^{\circ}\right.$ en diagonale, environ $20^{\circ}$ à l'horizontale pour les lunettes BT-300) limite la visibilité des menus virtuels et mixtes et influe sur la tâche de pointage [27]. L'impact de la taille du champ de vision augmenté nécessite d'être davantage étudié vis-à-vis de la physicalité des cibles.

$\mathrm{Au}$-delà des performances et des préférences, la physicalité de la représentation des widgets implique de considérer la flexibilité de l'interface utilisateur qui en résulte. Dans la littérature sur les interfaces utilisateur tangibles (TUI), la flexibilité est souvent déclinée selon deux aspects : le multiplexage temporel et le multiplexage spatial [13, 28, 43]. Le multiplexage temporel est basé sur un dispositif physique contrôlant différentes fonctions au cours du temps. Au contraire, le multiplexage spatial implique que chaque fonction a un dispositif physique dédié à une position spatiale donnée dans l'environnement [13]. Les TUI suivent une conception multiplexée dans l'espace, ce qui les rend intuitifs à utiliser [6]. Le multiplexage temporel et le multiplexage spatial font référence aux dispositifs d'entrée manipulés. Dans le cadre de notre étude, nous interrogeons les aspects de multiplexage temporel et spatial par rapport aux menus comme méta-instruments d'entrée [5].

Sur la base de ces concepts et des résultats de notre étude expérimentale, nous proposons un ensemble d'avantages et de limitations des menus virtuels, mixtes et physiques en RM. La figure 9 résume ces avantages et limitations pour chaque type de menu. Notre objectif est d'inspirer de nouvelles directions de conception qui peuvent être établies en considérant des applications particulières et des environnements écologiques.

\subsection{Menus virtuels}

Les menus virtuels permettent une entrée multiplexée dans le temps : ils peuvent changer leurs éléments (et donc, les commandes disponibles) au fil du temps. De plus, les menus virtuels permettent également un multiplexage spatial complet car ils ne reposent pas sur des objets physiques. Ils peuvent être placés à n'importe quelle position dans l'environnement sans créer d'encombrement physique. Les menus virtuels offrent ainsi une grande flexibilité de l'interface utilisateur. Cependant, aucun composant du menu n'est visible sans un casque de RM. Notre étude suggère également que l'utilisation des menus virtuels est l'approche la moins préférée et ne permet pas aux participants de transférer les compétences acquises à l'aide de menus virtuels vers d'autres types de menus.

\subsection{Menus mixtes}

Les menus mixtes proposent un bon compromis entre les menus virtuels et physiques. Ils permettent une entrée multiplexée dans le temps car les éléments virtuels attachés aux ancres physiques peuvent évoluer dans le temps. En termes de performances, nous n'avons pas trouvé de preuve de différence entre les menus mixtes et les menus virtuels. Ce résultat confirme celui de l'étude par Bailly et al [3]. Cependant, sur la base des questionnaires Raw-TLX, la plupart des participants ont préféré utiliser des menus mixtes plutôt que des menus virtuels. Ce résultat confirme également celui de Bailly et al [3], mais cette fois dans le cas d'une charge cognitive importante pendant les différentes tâches, comme en témoignent les questionnaires Raw-TLX. Néanmoins, la flexibilité de l'interface utilisateur des menus mixtes est limitée par leurs ancres physiques. Les menus mixtes ne prennent donc en charge qu'un multiplexage spatial limité dans le cadre de notre étude.

\subsection{Menus physiques}

Les résultats expérimentaux suggèrent que le fait de pouvoir voir le menu complet dans la vision périphérique peut avoir un impact positif clair sur les performances et les préférences. En outre, le transfert asymétrique des compétences entre les conditions observées dans cette étude ouvre des perspectives intéressantes pour la conception d'applications en RM. L'utilisation de menus physiques peut aider les utilisateurs à acquérir rapidement des compétences transférables sur l'interaction avec des menus via la tête. Par exemple, des menus physiques pourraient être utilisés lors de la familiarisation avec un nouveau système afin de permettre aux utilisateurs d'atteindre des performances optimales plus rapidement lorsqu'ils interagissent avec des menus virtuels. Il s'agit d'une considération importante pour des contextes spécifiques comme la chirurgie augmentée, où le temps est un facteur critique. Les chirurgiens doivent devenir efficaces avec un système le plus rapidement possible. La réduction du temps d'apprentissage pourrait ainsi favoriser l'adoption et le développement de systèmes de réalité augmentée pour la chirurgie.

Outre la capacité à voir l'ensemble du menu dans la vision périphérique, un autre facteur possible expliquant les bonnes performances des menus physiques pourrait être leur réalisme visuel. Dans leurs travaux, Jansen et al. ont observé qu'une visualisation 


\begin{tabular}{|c|c|c|}
\hline Physicalité du menu & Avantages & Limitations \\
\hline Menu virtuel & $\begin{array}{c}\text { Multiplexage temporel } \\
\text { Multiplexage spatial complet } \\
\text { Pas d'encombrement physique }\end{array}$ & $\begin{array}{l}\text { Casque nécessaire pour voir le menu virtuel } \\
\text { Ne peut pas bénéficier du monde physique } \\
\text { Plus lent, moins apprécié que les deux autres }\end{array}$ \\
\hline Menu mixte & $\begin{array}{c}\text { Multiplexage temporel } \\
\text { Préféré aux menus virtuels }\end{array}$ & $\begin{array}{l}\text { Casque nécessaire pour voir les éléments du menu } \\
\text { Multiplexage spatial limité aux ancres physiques } \\
\text { Mêmes performances que les menus virtuels }\end{array}$ \\
\hline Menu physique & $\begin{array}{c}\text { Meilleure performance } \\
\text { Type de menu préféré } \\
\text { Favorise l'apprentissage (transfert de compétence) }\end{array}$ & $\begin{array}{l}\text { Pas de multiplexage spatial ni temporel } \\
\text { Pas de mobilité } \\
\text { Coût de mise en place et de mise à jour élevé }\end{array}$ \\
\hline
\end{tabular}

Figure 9: Récapitulatif des avantages et limitations des menus virtuels, mixtes et physiques.

3D physique conduisait à de meilleures performances qu'une visualisation à l'écran lors d'une tâche de recherche d'informations [26]. Les auteurs suggèrent que le réalisme visuel de la visualisation physique peut avoir un impact positif sur les performances d'interaction. Cependant, dans notre étude les participants devaient effectuer des tâches de pointage 2D et non des tâches de recherche d'informations sur des visualisations 3D. Nous avons visé dans notre étude à concevoir des menus autant que possible visuellement similaires comme le montre la Figure 4. Néanmoins, les indices visuels tels que les indices stéréoscopiques, d'accommodation et de texture nécessitent de plus amples études pour évaluer leur impact sur l'interaction avec des menus en RM.

Par ailleurs, les menus physiques reposent entièrement sur des objets physiques et ne permettent pas une entrée multiplexée dans le temps ni dans l'espace. En outre, l'introduction d'objets physiques dans des environnements restreints peut être gênante. Même lorsque cela est possible, les utilisateurs peuvent ne pas avoir le contrôle sur la position des menus physiques. Ces derniers sont également coûteux à créer et à mettre à jour. Par conséquent, les bonnes performances et les propriétés des menus physiques ont un coût non négligeable.

Enfin, nous nous sommes concentrés dans cette étude sur l'aspect perceptuel de la physicalité des menus. Il serait intéressant de poursuivre ces travaux en s'intéressant aux aspects cognitifs, dont la mémorisation des menus et des commandes. Ainsi, créer des liens sémantiques entre objets physiques et numériques pourrait aider les utilisateurs à apprendre l'organisation de l'environnement mixte [14]. Par exemple pour le cas de la chirurgie augmentée ancrer un menu lié à la visibilité des objets virtuels sur la caméra du bloc opératoire (Figure 2).

\section{CONCLUSION}

Cet article contribue à l'interaction basée sur les mouvements de la tête avec des menus en RM avec un casque. Motivé par le contexte applicatif de la chirurgie augmentée et inspiré par l'espace de conception proposé par Bailly et al. [3], l'étude présentée est dédiée à l'impact de la physicalité des menus. Nous contribuons ainsi à explorer une nouvelle dimension des widgets.

Lors d'une étude expérimentale, nous avons comparé des menus virtuels, mixtes et physiques et étudié leur influence sur les performances des tâches de pointage avec des mouvements de la tête. Les résultats expérimentaux suggèrent que les menus physiques surpassent les menus mixtes et virtuels et sont largement préférés par les participants. Par ailleurs, seuls les menus physiques ont permis le transfert de compétences vers d'autres types de menus. Un tel résultat ouvre des perspectives prometteuses pour se familiariser avec un environnement mixte. Au contraire, nous n'avons observé aucune différence de performances entre les menus mixtes et virtuels, même si les menus mixtes ont été préférés. Enfin, nous proposons un ensemble d'avantages et de limites de menus virtuels, mixtes et physiques pour inspirer les concepteurs et les études futures.

Grâce à un partenariat industriel, nous avons implémenté des menus virtuels, mixtes et physiques dans un système de navigation chirurgical professionnel. Nous envisageons de mener des études dans des environnements pseudo-écologiques (salle d'opération, pédale de commande pour confirmer une sélection) avec des professionnels du domaine et des chirurgiens. Nous envisageons également d'étudier une modélisation du pointage en deux phases (pointer vers le menu, puis vers l'élément) : 1) avec des menus mixtes ou virtuels en utilisant des distances angulaires [46] et 2) en comparant ce modèle à celui d'un pointage classique pour des menus physiques.

\section{RÉFÉRENCES}

[1] Aesculap. 2020. OrthoPilot ${ }^{\circledR}$ navigation system. Retrieved June 9th, 2020 from https://www.bbraun.com/en/products-and-therapies/orthopaedic-jointreplacement/orthopilot.html/orthopilot.html

[2] Takumi Azai, Shuhei Ogawa, Mai Otsuki, Fumihisa Shibata, and Asako Kimura. 2017. Selection and Manipulation Methods for a Menu Widget on the Human Forearm. In Proceedings of the 2017 CHI Conference Extended Abstracts on Human Factors in Computing Systems (Denver, Colorado, USA) (CHI EA '17). Association for Computing Machinery, New York, NY, USA, 357-360.

[3] Charles Bailly, François Leitner, and Laurence Nigay. 2019. Head-Controlled Menu in Mixed Reality with a HMD. In Human-Computer Interaction - INTERACT 2019. Springer International Publishing, 395-415.

[4] Gilles Bailly, Eric Lecolinet, and Laurence Nigay. 2016. Visual Menu Techniques. ACM Comput. Surv. 49, 4, Article 60 (Dec. 2016), 41 pages.

[5] Michel Beaudouin-Lafon. 2000. Instrumental interaction : An Interaction Model for Designing Post-WIMP User Interfaces. Proc. 18th Int. Conf. Hum. factors Comput. Syst. - CHI '00 2, 1 (2000), 446-453.

[6] Mark Billinghurst, Raphael Grasset, and Julian Looser. 2005. Designing augmented reality interfaces. ACM Siggraph Computer Graphics 39, 1 (2005), 17-22.

[7] Mark Billinghurst, Hirokazu Kato, Ivan Poupyrev, et al. 2008. Tangible augmented reality. ACM SIGGRAPH ASIA 7, 2 (2008), 1-10.

[8] Siyuan Chen, Julien Epps, and Fang Chen. 2011. A comparison of four methods for cognitive load measurement. In Proc. 23rd Aust. Comput. Interact. Conf. OzCHI '11. https://doi.org/10.1145/2071536.2071547

[9] Andy Cockburn. 2019. Anchoring Effects and Troublesome Asymmetric Transfer in Subjective Ratings. CHI 2019 (2019), 1-12.

[10] Pierre Dragicevic, Fair Statistical, H C I Modern, and Statistical Methods. 2016. Fair Statistical Communication in HCI Pierre Dragicevic To cite this version : Fair Statistical Communication in HCI. 291-330 pages. 
[11] Andrew T Duchowski, Krzysztof Krejtz, Izabela Krejtz, Cezary Biele, Anna Niedzielska, Peter Kiefer, Martin Raubal, and Ioannis Giannopoulos. 2018. The index of pupillary activity : measuring cognitive load vis-à-vis task difficulty with pupil oscillation. In Proceedings of the 2018 CHI Conference on Human Factors in Computing Systems. ACM, New York, NY, USA, 282.

[12] Andéol Evain, Ferran Argelaguet, Nicolas Roussel, Géry Casiez, and Anatole Lécuyer. 2017. Can i think of something else when using a bci? : Cognitive demand of an ssvep-based bci. In Proceedings of the 2017 CHI Conference on Human Factors in Computing Systems. ACM, New York, NY, USA, 5120-5125.

[13] George W. Fitzmaurice and William Buxton. 1997. Empirical evaluation of graspable user interfaces : Towards specialized, space-multiplexed input. Conf. Hum. Factors Comput. Syst. - Proc. 1 (1997), 43-50.

[14] Bruno Fruchard, Eric Lecolinet, and Olivier Chapuis. 2018. Impact of semantic aids on command memorization for on-body interaction and directional gestures. In Proceedings of the 2018 International Conference on Advanced Visual Interfaces. Association for Computing Machinery, New York, NY, USA, 1-9.

[15] Anthony G Greenwald. 1976. Within-subjects designs : To use or not to use? Psychological Bulletin 83, 2 (1976), 314.

[16] Maxime Guillon, François Leitner, and Laurence Nigay. 2014. Static VoronoiBased Target Expansion Technique for Distant Pointing. In Proceedings of the 2014 International Working Conference on Advanced Visual Interfaces (Como, Italy) (AVI '14). Association for Computing Machinery, New York, NY, USA, 41-48.

[17] Eija Haapalainen, Seungjun Kim, Jodi F Forlizzi, and Anind K Dey. 2010. PsychoPhysiological Measures for Assessing Cognitive Load.

[18] Sandra G. Hart and Lowell E. Staveland. 1988. Development of NASA-TLX (Task Load Index) : Results of Empirical and Theoretical Research. Adv. Psychol. 52, C (1988), 139-183. https://doi.org/10.1016/S0166-4115(08)62386-9

[19] Steven J Henderson and Steven Feiner. 2009. Evaluating the benefits of augmented reality for task localization in maintenance of an armored personnel carrier turret. In 2009 8th IEEE International Symposium on Mixed and Augmented Reality. IEEE, 135-144.

[20] Niels Henze and Susanne Boll. 2010. Evaluation of an off-screen visualization for magic lens and dynamic peephole interfaces. Proc. 12th Int. Conf. Hum. Comput. Interact. with Mob. devices Serv. - MobileHCI '10 (2010), 191. https: //doi.org/10.1145/1851600.1851632

[21] David M. Hoffman, Ahna R. Girshick, Kurt Akeley, and Martin S. Banks. 2008 Vergence-accommodation conflicts hinder visual performance and cause visual fatigue. Journal of Vision 8, 3 (2008), 33.

[22] Shamsi T. Iqbal, Xianjun Sam Zheng, and Brian P. Bailey. 2004. Task-evoked pupillary response to mental workload in human-computer interaction. In $\mathrm{CHI}$ 2004. 1477. https://doi.org/10.1145/985921.986094

[23] Hiroshi Ishii. 2008. The tangible user interface and its evolution. Commun. ACM 51, 6 (2008), 32-36.

[24] ISO9241-9:2000(E) 2000. Ergonomic requirements for office work with visual display terminals (VDTs) - Part 9. Standard. International Organization for Standardization.

[25] Shahram Jalaliniya, Diako Mardanbeigi, Thomas Pederson, and Dan Witzner Hansen. 2014. Head and eye movement as pointing modalities for eyewear computers. Proc. - 11th Int. Conf. Wearable Implant. Body Sens. Networks Work. BSN Work. 2014 (2014), 50-53. https://doi.org/10.1109/BSN.Workshops.2014.14

[26] Yvonne Jansen, Pierre Dragicevic, and Jean-Daniel Fekete. 2013. Evaluating the efficiency of physical visualizations. (2013), 2593. https://doi.org/10.1145/ 2470654.2481359

[27] Bonifaz Kaufmann and David Ahlström. 2012. Revisiting Peephole Pointing : A Study of Target Acquisition with a Handheld Projector. MobileHCI'12 (2012), 211-220.

[28] Hyunyoung Kim, Céline Coutrix, and Anne Roudaut. 2018. Knobslider : Design of a shape-changing UI for parameter control. Conf. Hum. Factors Comput. Syst. Proc. 2018-April (2018). https://doi.org/10.1145/3173574.3173913

[29] Ernst Kruijff, J Edward Swan, and Steven Feiner. 2010. Perceptual issues in augmented reality revisited. In 2010 IEEE International Symposium on Mixed and Augmented Reality. IEEE, 3-12.

[30] Mikko Kytö, Barrett Ens, Thammathip Piumsomboon, Gun A Lee, and Mark Billinghurst. 2018. Pinpointing : Precise Head - and Eye - Based Target Selection for Augmented Reality. Chi18 April (2018), 1-14. https://doi.org/10.1145/3173574. 3173655

[31] Hyeongmook Lee and Woontack Woo. 2010. Tangible spin cube for 3D ring menu in real space. In Proc. 28th Int. Conf. Ext. Abstr. Hum. factors Comput. Syst. CHI EA '10. 4147. https://doi.org/10.1145/1753846.1754117

[32] Anna Macaranas, Alissa N. Antle, and Bernhard E. Riecke. 2012. Bridging the Gap : Attribute and Spatial Metaphors for Tangible Interface Design. In Proceedings of the Sixth International Conference on Tangible, Embedded and Embodied Interaction (Kingston, Ontario, Canada) (TEI '12). Association for Computing Machinery, New York, NY, USA, 161-168.

[33] I Scott MacKenzie. 2012. Human-computer interaction : An empirical research perspective. Newnes.

[34] David E. Meyer, Richard A. Abrams, Sylvan Kornblum, Charles E. Wright, and J. E.Keith Smith. 1988. Optimality in Human Motor Performance : Ideal Control of Rapid Aimed Movements. Psychol. Rev. 95, 3 (1988), 340-370. https://doi.org/ 10.1037/0033-295X.95.3.340

[35] Microsoft. 2020. Available cursors for Hololens 1 and 2. Retrieved October 2020 from https://docs.microsoftcom/en-us/windows/mixed-reality/design/cursors

[36] Paul Milgram and Fumio Kishino. 1994. A taxonomy of mixed reality visual displays. IEICE TRANSACTIONS on Information and Systems 77, 12 (1994), 13211329.

[37] S. K. Ong, M. L. Yuan, and A. Y.C. Nee. 2008. Augmented reality applications in manufacturing : A survey. Int. F. Prod. Res. 46, 10 (2008), 2707-2742.

[38] Patrick Perea, Denis Morand, and Laurence Nigay. 2019. Spotlight on Off-Screen Points of Interest in Handheld Augmented Reality : Halo-Based Techniques. In Proceedings of the 2019 ACM International Conference on Interactive Surfaces and Spaces (Daejeon, Republic of Korea) (ISS '19). Association for Computing Machinery, New York, NY, USA, 43-54.

[39] Michael Rohs and Antti Oulasvirta. 2008. Target acquisition with camera phones when used as magic lenses. Proceeding twenty-sixth Annu. CHI Conf. Hum. factors Comput. Syst. - CHI '08 (2008), 1409.

[40] R. William Soukoreff and I. Scott MacKenzie. 2004. Towards a standard for pointing device evaluation, perspectives on 27 years of Fitts' law research in HCI. Int. F. Hum. Comput. Stud. 61, 6 (2004), 751-789. https://doi.org/10.1016/j.ijhcs. 2004.09.001

[41] Maximilian Speicher, Brian D. Hall, and Michael Nebeling. 2019. What is Mixed Reality?. In Proceedings of the 2019 CHI Conference on Human Factors in Computing Systems (Glasgow, Scotland Uk) (CHI '19). Association for Computing Machinery, New York, NY, USA, 1-15.

[42] Alvin R Tilley et al. 2001. The measure of man and woman : human factors in design. John Wiley \& Sons.

[43] Philip Tuddenham, David Kirk, and Shahram Izadi. 2010. Graspables revisited : Multi-touch vs. tangible input for tabletop displays in acquisition and manipulation tasks. Conf. Hum. Factors Comput. Syst. - Proc. 4 (2010), 2223-2232. https://doi.org/10.1145/1753326.1753662

[44] Brygg Ullmer, Urban Wiggins, Shining Sun, Rajesh Sankaran, Srikanth Jandhyala, Blake Tregre, Cornelius Toole, Karun Kallakuri, Christopher Laan, Matthew Hess, and Farid Harhad. 2008. Tangible menus and interaction trays. In Proc. 2nd Int. Conf. Tangible Embed. Interact. - TEI '08. 209. https://doi.org/10.1145/1347390. 1347436

[45] Sean White, David Feng, and Steven Feiner. 2009. Interaction and presentation techniques for shake menus in tangible augmented reality. Sci. Technol. Proc. IEEE 2009 Int. Symp. Mix. Augment. Reality, ISMAR 2009 July (2009), 39-48.

[46] Chadwick A Wingrave and Doug A Bowman. 2004. Baseline Factors for Raycasting Selection. Virtual Real. Int. (2004). 